\title{
The origin of trimethylamine- $N$-oxide (TMAO) and its role in development of atherosclerosis
}

\author{
Zouyan He and Zhen-Yu Chen*
}

Food \& Nutritional Sciences Programme, School of Life Sciences, The Chinese University of Hong Kong, Shatin, NT, Hong Kong, China *Corresponding author: Zhen-Yu Chen, School of Life Sciences, The Chinese University of Hong Kong, Shatin, NT, Hong Kong, China. Tel: (852) 3943-6382; Fax: (852) 2603-7246; E-mail: zhenyuchen@cuhk.edu.hk

DOI: $10.31665 / J F B .2018 .2138$

Received: April 05, 2018; Revised received \& accepted: May 22, 2018

Citation: He, Z., and Chen, Z.-Y. (2018). The origin of trimethylamine- $N$-oxide (TMAO) and its role in development of atherosclerosis. J. Food Bioact. 2: 28-36.

\begin{abstract}
Cardiovascular disease (CVD) is a leading cause of deaths and is a growing epidemic worldwide. Atherosclerosis, the primary cause of heart diseases and stroke, is associated with vascular inflammation and accumulation of lipids and fibrous elements in the arteries. Recently, blood trimethylamine- $N$-oxide (TMAO) has been identified as an independent risk factor for CVD in humans. TMAO is mainly derived from dietary trimethylamine (TMA)-containing nutrients via the bioconversion of gut microbiota and hepatic flavin monooxygenases (FMOs). Both in vivo and in vitro studies have revealed that TMAO promotes atherogenesis by exacerbating vascular inflammation, impairing vascular functions and disturbing cholesterol homeostasis at multiple levels. This review summarizes the current research on the microbiota-dependent generation pathway of TMAO, the associations of TMAO with atherosclerosis, and the potential dietary interventions to reduce the TMAO-associated risk of CVD.
\end{abstract}

Keywords: Cardiovascular diseases; Inflammation; Flavin monooxygenases; Trimethylamine- $N$-oxide; trimethylamine.

\section{Introduction}

Cardiovascular disease (CVD) has become an evolving epidemic and is the number one killer in the world (Benjamin et al. 2017). Most CVD can be attributable to atherosclerosis, which is characterized by narrowing or blockage of large arteries (Lusis 2000). Dyslipidemia, cigarette smoking, hypertension, obesity, diabetes as well as family history have been well-recognized as the major risk factors for CVD (Wilson et al. 1998). Recently, trimethylamine- $N$ oxide (TMAO), a microbial-derived metabolite from dietary nutrients, has also been identified as an independent risk factor for atherosclerosis (Wang et al. 2011). TMAO, which links diet, gut microbiota, and host metabolism to development of atherosclerosis and other CVD, has been a hot research focus in this decade (He et al. 2017). This review aims to discuss (i) the microbiota-dependent production of TMAO from dietary sources, (ii) the pathological activity of TMAO in the development of atherosclerosis and CVD, and (iii) the possible dietary intervention strategies for the prevention and treatment of TMAO-related atherogenesis.

\section{Origin and metabolism of TMAO}

TMAO is a colorless and odorless amine oxide with a molecular mass of 75.1 Da (Velasquez et al. 2016). It is known as an osmoregulatory compound in marine fish to adjust buoyancy, protect against the adverse environmental stress in deep ocean, and stabilize protein structure (Withers et al. 1994; Gillett et al. 1997; Yancey 2005). In humans, a positive correlation has been found between elevated blood TMAO levels and increased risk in CVD (Wang et al. 2011; Tang et al. 2013). Most TMAO in blood is derived from dietary trimethylamine (TMA)-containing nutrients including choline, phosphatidylcholine, L-carnitine and betaine (Wang et al. 2011; Koeth et al. 2013). After ingestion, gut microbes liberate the TMA moiety from the TMA-containing nutrients in the intestine. Most TMA in the intestine is passively absorbed into portal circulation and is then oxidized to produce TMAO by flavin monooxygenases (FMOs) in the liver (Zeisel and Warrier 2017). In humans, blood TMAO is mainly eliminated through urine as well as sweat and breath (Bain et al. 2005; Taesuwan et al. 2017) 


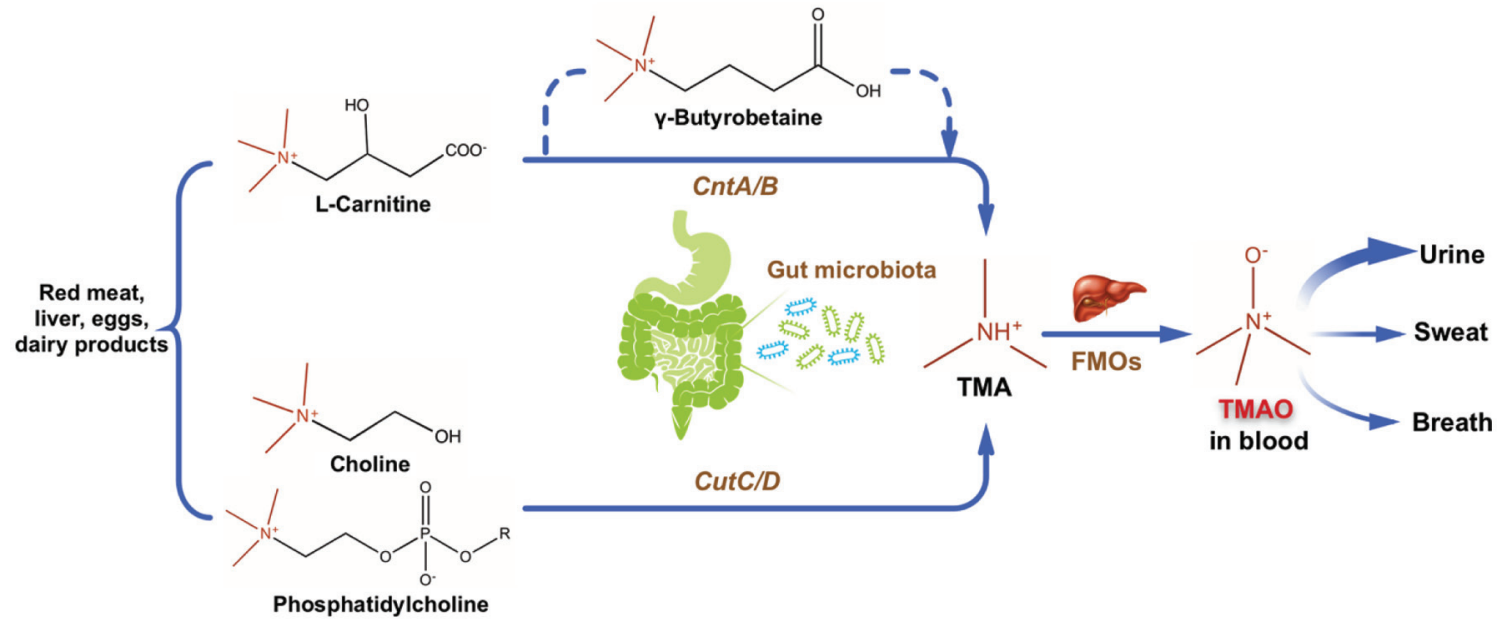

Figure 1. Production of TMAO from dietary TMA-containing components and metabolism of TMAO. TMA, trimethylamine; TMAO, trimethylamine- $N$ oxide; FMOs, flavin monooxygenases. CntA/B, two-component Rieske-type oxygenase/reductase; CutC/D, choline TMA-lyase and its activating enzyme.

(Figure 1).

\subsection{Dietary sources of TMAO}

TMA-containing nutrients exist in a wide range of foods such as red meat, liver, eggs and dairy products and are the primary precursors of blood TMAO. It has been reported that administration of 8-oz sirloin steak (containing estimated $180 \mathrm{mg}$ of L-carnitine) or two hard-boiled eggs with yolk (each containing approximately $250 \mathrm{mg}$ of choline) could increase plasma TMAO levels when the intact gut microbiota is present (Tang et al. 2013; Koeth et al. 2013). Consumption of more than two eggs could raise both plasma and urine TMAO concentrations with about $14 \%$ of choline in eggs being converted to TMAO (Miller et al. 2014). Oral administration of $\gamma$-butyrobetaine, a metabolite from dietary L-carnitine, was reported to raise plasma TMA and TMAO concentrations in mice in a microbiota-dependent manner (Koeth et al. 2014).

Marine fish is another notable dietary source of TMAO. The TMAO content in the muscles of marine teleosts, skates, shrimps and craps is $22-299 \mathrm{mmol} / \mathrm{kg}(1.7-22.5 \mathrm{~g} / \mathrm{kg})$, which positively correlated with the habitat depth of these marine animals (Kelly and Yancey 1999). A similar amount of approximate 40-200 mmol of TMAO $(3-15 \mathrm{~g})$ is detected in $1 \mathrm{~kg}$ of white muscle of seven teleosts (Treberg and Driedzic 2002). Svensson et al. (1994) showed that urinary TMA and TMAO levels reflected positively the recent fish intake. Moreover, after a 4-week dietary intervention in which $60 \%$ of the protein was substituted with lean-seafood, concentrations of TMAO was raised by 5.5 folds in urine, and by 2 folds in fasting serum of 20 healthy subjects (Schmedes et al. 2016). In a crossover trial involving 40 healthy young men, fish consumption yielded a greater increase in blood TMAO levels than either eggs or beef (Cho et al. 2017). However, Rohrmann et al. (2016) observed no obvious effect of dietary intake of meat, egg or fish on plasma TMAO levels, but a positive association between dairy food consumption and plasma TMAO concentrations in 271 participants.

\subsection{Intestinal microbiota-dependent production of TMAO}

Gut microbiota consist of trillions of commensal microorganisms residing in human intestine and play an obligatory role in the liberation of TMA moiety from dietary TMA-containing nutrients. Oral administration of broad-spectrum antibiotics abolished the production of TMAO from deuterium labeled phosphatidylcholine (d9-PC) because antibiotics inhibited the growth of gut bacteria in mice (Wang et al. 2011). Recolonization of gut microbes recovered the generation of TMAO after oral d9-PC treatment (Wang et al. 2011). Similar studies were conducted for d9-PC and deuterium labeled L-carnitine in healthy participants (Tang et al. 2013; Koeth et al. 2013), confirming the production of TMAO from dietary precursors is in a microbiota-dependent manner.

Gut microbial composition directly determines the TMA-generating capacity from dietary TMA-containing precursors. In the study of Koeth et al. (2013), individuals with an enterotype rich in the Prevotella genus had higher plasma TMAO concentrations after L-carnitine intake than did subjects with an enterotype rich in the Bacteroidetes genus. Nine strains of bacteria from human intestinal isolates, which represents two different phyla (Firmicutes and Proteobacteria) and six genera, were identified to produce TMA from choline in vitro (Romano et al. 2015). Moreover, it was reported that high-TMAO producers had a less diverse gut microbiome and a higher Firmicutes-to-Bacteroidetes ratio (around 2:1), than those of low-TMAO producers whose Firmicutes-toBacteroidetes ratio was about 1:1 (Cho et al. 2017). In contrast, in mice with an oral administration of L-carnitine, plasma TMAO level was found to be positively correlated with Bacteroidetes and negatively correlated with Firmicutes (Zhao et al. 2018). In addition, two clusters of genes $\mathrm{CutC} / \mathrm{D}$ and $\mathrm{CntA} / \mathrm{B}$, were identified as the pivotal genes for the microbial production of TMAO from choline and L-carnitine, respectively (Craciun and Balskus 2012; Zhu et al. 2014). Therefore, modulating the intestinal microbial community or targeting at the microbial genes related to TMA production may become a potential therapeutic approach to prevent the TMAO-associated CVD.

\subsection{Conversion of TMA to TMAO by flavin monooxygenas- es (FMOs) in the liver}

Hepatic oxidation catalyzed by FMOs is necessary for the conversion of TMA to TMAO. Six functional FMOs (FMO1, 2, 3, 4, 5 and 6) have been identified in humans (Hernandez et al. 2004). 
In human liver, FMO3 is the major adult isoform of FMOs and its expression switches on after birth (Koukouritaki et al. 2002). Interestingly, females have a higher hepatic FMO3 expression than males (Bennett et al. 2013). Veervalli et al. (2017) reported that the most amount of TMAO was produced by hepatic FMO3 in female mice. In contrast, most amount of TMAO was derived from the action of FMO1 in male mice which lack hepatic FMO3. This sexual dimorphism of FMO3 expression was affected by sexual hormones based on the observations that androgen showed repressive while estrogen exhibited inductive effects on FMO3 expression in mice (Bennett et al. 2013). Defect in hepatic FMO3 expression results in trimethylaminuria or fish-odor syndrome, due to excessive accumulation of the odorous TMA which could be secreted in the urine, breath or sweat (Dolphin et al. 1997). Apart from its critical role in TMAO production, FMO3 was shown to be involved in the modulation of glucose and lipid homeostasis, hepatic cholesterol and triacylglycerol metabolism, and development of obesity (Shih et al. 2015; Warrier et al. 2015; Schugar et al. 2017).

\section{High correlation between circulating TMAO and CVD in hu- mans}

A positive correlation between circulating TMAO and CVD risk was first revealed in the study of Wang et al. (2011). By using untargeted metabolomics analyses, plasma TMAO, choline and betaine were identified as markers to predict the increased risk for CVD in humans in an initial learning cohort and a subsequent independent validation cohort (Wang et al. 2011). These three molecules showed dose-dependent associations with CVD in a larger independent validation cohort study $(\mathrm{N}=1,876)$ (Wang et al. 2011). Since then, the relationship between blood TMAO levels and risk of CVD has become an emerging area of research.

Consistent with the findings of Wang et al. (2011), many studies have reported that elevated blood TMAO concentration is associated with increased CVD risk. In a 3-year cohort study involving 4,007 patients undergone coronary angiography, plasma TMAO levels were found to be positively correlated with the risk of incident major adverse cardiovascular events (MACE) including myocardial infarction, stroke, and death (Tang et al. 2013). A similar positive correlation was observed between high plasma levels of choline and betaine with incident MACE risk in another 3-year follow-up in 3,903 subjects with elective diagnostic coronary angiography (Wang et al. 2014). However, this correlation existed only when increased plasma TMAO was present (Wang et al. 2014). In a multiethnic population-based study with 99 CVD cases and 193 unmatched control individuals, plasma TMAO concentrations significantly associated with prevalent CVD, even after adjusted for diabetes status, meat, fish and cholesterol intake (Mente et al. 2015). But no significant association was found between plasma L-carnitine levels and prevalent CVD (Mente et al. 2015). Besides, elevated plasma TMAO levels were also observed in patients with chronic heart failure and were associated with advanced left ventricular diastolic dysfunction and poorer long-term adverse clinical outcomes (Tang et al. 2015b; Trøseid et al. 2015). In patients with coronary artery disease, increased plasma TMAO levels were reported to be strongly associated with heavier atherosclerotic burden and higher long-term mortality risk (Senthong et al. 2016a, 2016b). Moreover, high plasma betaine and TMAO concentrations were shown to be markers of cardiovascular risk in diabetic patients, whereas low plasma betaine concentrations suggested the increased risk of CVD in the absence of diabetes (Lever et al. 2014). Similarly, it was reported that increased serum TMAO levels correlated with increased carotid intima-media thickness (cIMT), an early hallmark of atherosclerosis, independent of conventional cardiovascular risk markers including insulin resistance, visceral obesity and fatty liver in subjects at risk for type II diabetes (Randrianarisoa et al. 2016). Furthermore, Zheng et al. (2016) observed that higher dietary consumption of phosphatidylcholine was associated with increased all-cause and CVD mortality in the US population, especially for diabetic patients.

Nevertheless, controversial results regarding the correlation between dietary intake of TMA-containing components and CVD risk were reported in some other studies. In a study involving 14,430 middle-aged men and women, no association was found between dietary choline intake and incident coronary heart disease (Bidulescu et al. 2007). A meta-analysis of 13 controlled trials (N $=3,629)$ revealed that compared with placebo or control, oral Lcarnitine intake was associated with a $27 \%$ reduction in all-cause mortality, a $65 \%$ reduction in ventricular arrhythmias and a $40 \%$ reduction in angina symptoms in patients experiencing an acute myocardial infarction (DiNicolantonio et al. 2013). Consistently, although an oral L-carnitine supplement $(900 \mathrm{mg} / \mathrm{d})$ raised plasma TMA and TMAO levels, it lowered the plasma markers of vascular injury and oxidative stress, exhibiting vasculoprotective activity in hemodialysis patients (Fukami et al. 2015). In addition, among 3,942 African-American participants in Jackson Heart Study, dietary choline intake was negatively correlated with the incidence of ischemic stroke, while higher dietary betaine intake was associated with a nonlinear higher risk of the incidence of coronary heart disease (Millard et al. 2016).

Plasma TMAO may also correlate with some other diseases. Patients suffering from chronic kidney disease (CKD) with elevated plasma TMAO levels tended to have a higher risk for all-cause mortality (Tang et al. 2015a). In patients who have undergone a cardiovascular surgery, a higher serum TMAO concentration was associated with advanced CKD stages as well as increased number of infarcted coronary arteries (Mafune et al. 2016). Additionally, plasma TMAO was reported to be positively correlated with colorectal cancer risk among postmenopausal women (Bae et al. 2014).

\section{Effects of TMAO on atherogenesis and plasma cholesterol}

The causal relationship between plasma TMAO concentrations and atherosclerosis was examined in animal models. Dietary TMAO, L-carinitine or choline supplements were shown to facilitate atherogenesis with the formation of larger atherosclerotic plaques in mice in many studies (Wang et al. 2011; Koeth et al. 2013; Chen et al. 2016, 2017; Geng et al. 2017; Zhao et al. 2018). Even though TMAO-induced atherosclerosis has been investigated both in vitro and in vivo, the pathogenic mechanism still remains poorly understood. It is believed that aggravated vascular inflammation, impairments in blood vessels and disturbance in cholesterol homeostasis may contribute to the TMAO-induced atherogenesis (Figure 2).

\subsection{TMAO-induced vascular inflammation}

Atherosclerosis is a complex inflammatory disease characterized by accumulation of fat inside the arterial lumen (Lusis 2000). Vascular inflammation may damage the arterial intima, accelerating the progression of atherogenesis (Ross 1999). Studies have shown that TMAO is able to aggravate vascular inflammation. In the research of Seldin et al. (2016), elevation of inflammatory gene expression was observed in the aortas of $\mathrm{LDLR}^{-/-}$mice supplemented with 


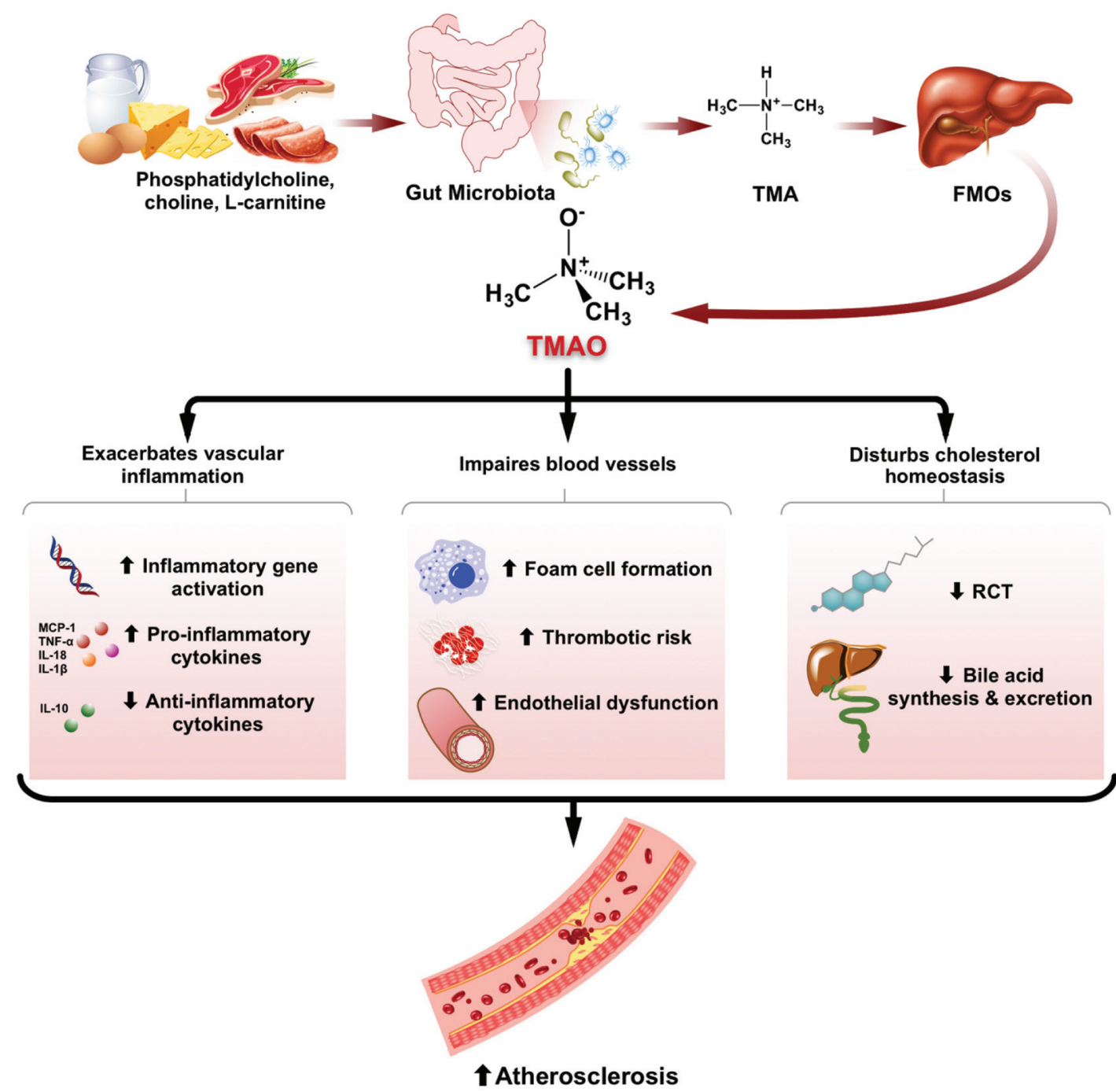

Figure 2. Effects of microbiota-dependent TMAO production on development of atherosclerosis. TMA, trimethylamine; TMAO, trimethylamine- $\mathrm{N}$-oxide; FMOs, flavin monooxygenases; RCT, reverse cholesterol transport.

$1.3 \%$ of choline in drinking water. Acute intraperitoneal injection of TMAO at physiological levels induced the same inflammatory markers and activated the phosphorylation of $\mathrm{p} 38$ mitogen-activated protein kinase (MAPK), extracellular signal-kinase 1/2 (ERK $1 / 2)$ and p65 nuclear factor- $\mathrm{KB}(\mathrm{NF}-\mathrm{\kappa B})$ (Seldin et al. 2016), all of which play important roles in cellular inflammation and contributing to atherogenesis (Baker et al. 2011). These observations were recapitulated in human aortic endothelial cells (HAECs) and human vascular smooth muscle cells (Seldin et al. 2016). In addition, TMAO could induce inflammation in human umbilical vein endothelial cells (HUVECs) and aortas from $\mathrm{ApoE}^{-1-}$ mice by activating the NLRP3 inflammasome, which was in part mediated through SIRT3-SOD2-mitochondrial ROS signaling pathway (Chen et al. 2017). Similar activation of NLRP3 inflammasome and down-stream IL-1 $\beta$ production was observed both in TMAOtreated carotid artery endothelial cells (CAECs) and in TMAOinfused C57BL/6J mice with partially ligated carotid artery (Boini et al. 2017). Extensive inflammation was exacerbated by TMAO with evidence of elevations in pro-inflammatory gene expression and a decline in anti-inflammatory cytokine IL-10 expression in both serum and adipose tissue of high-fat-fed C57BL/6J mice supplemented with $0.2 \%$ of dietary TMAO (Gao et al. 2014, 2015).

\subsection{TMAO-induced endothelial dysfunction}

TMAO is also able to exert some detrimental effects on blood vessels. In the early stage of atherosclerosis, macrophages uptake the oxidized cholesterol mediated by scavenger receptors, leading to subendothelial accumulations of cholesterol-engorged macrophages, so called "foam cells" (Lusis 2000). TMAO was reported to accelerate the foam cell formation in some studies. Increased expressions of inflammatory adhesion-related genes including Eselectin, monocyte chemotactic protein 1 (MCP-1), intracellular adhesion molecule 1 (ICAM-1), and vascular cell adhesion molecule 1 (VCAM-1) were observed in the aorta of high-choline-fed $\mathrm{LDLR}^{-/-}$mice, promoting the recruitment of monocytes and their differentiation into macrophages (Seldin et al. 2016). Similarly, TMAO decreased the self-repair capacity and induced monocyte adhesion in HUVECs, which was partially contributed by the acti- 
vation of PKC/NF-kB/VCAM-1 pathway (Ma et al. 2017). Besides, TMAO was reported to up-regulate the expression of macrophage receptors (CD36 and SR-A1) in $\mathrm{ApoE}^{-/-}$mice fed a high-choline or high-TMAO diet (Wang et al. 2011), as well as in TMAO-treated murine macrophage J774A.1 cells (Mohammadi et al. 2016), facilitating the up-take of cholesterol into macrophage and foam cell formation. Subsequently, Geng et al. (2017) observed accelerated atherosclerotic plaque progression, promoted macrophage recruitment, higher CD36 and pro-inflammatory cytokine expression in the plaque lesions in high-TMAO-fed $\mathrm{ApoE}^{-/-}$mice. They also found that TMAO-related foam cell formation was mediated, at least partially, by the CD36/MAPK/JNK pathway (Geng et al. 2017). However, in murine J447 macrophage treated with acetylated-LDL, TMAO in increasing concentrations did not alter the cholesterol uptake or efflux (Collins et al. 2016).

TMAO impairs blood vessels by augmenting thrombotic risk and causing endothelial dysfunction. It was shown that TMAO directly enhanced platelet responsiveness in mice intraperitoneally injected with TMAO or administrated with a high-choline or a high-TMAO diet, resulting in increased thrombosis potential (Zhu et al. 2016). Moreover, endothelial dysfunction was induced by TMAO with an increase in the levels of serum vasoconstrictors endothelin 1 and thromboxane $\mathrm{A}_{2}$, while a decrease in the levels of serum vasodilators nitric oxide (NO) and prostaglandin $\mathrm{I}_{2}$ in high-TMAO- or high-choline-fed Kunming mice (Hu et al. 2015; Ren et al. 2016). Furthermore, TMAO treatment decreased the expression of inflammasome-dependent tight junction protein $\mathrm{ZO}-1$ in endothelial cells, leading to endothelial hyperpermeability and dysfunction (Boini et al. 2017). In a senescence-accelerated mouse model, TMAO raised the expression of vascular aging-related markers, accelerated aging-related vascular remodeling and aggravated vascular dysfunction by significantly decreasing endothelialdependent dilation capacity while increasing vascular contraction activity (Ke et al. 2018).

\subsection{Effect of TMAO on cholesterol homeostasis}

The pathogenesis of atherosclerosis is highly related to cholesterol metabolism. Research has shown that TMAO is able to disturb cholesterol homeostasis at multiple levels, including reverse cholesterol transport, de novo bile acid synthesis and excretion. In the study of Koeth et al. (2013), addition of TMAO in diet significantly reduced the reverse cholesterol transport (RCT) in $\mathrm{ApoE}^{-/}$ mice by $35 \%$. RCT is the net movement of cellular cholesterol from peripheral tissues to the liver for its excretion in the bile and ultimately the feces. Impairment of RCT is believed to aggravate the development of atherosclerosis (Rader et al. 2009). ATP cassette transporter type 1 (ABCA1) is to remove cellular cholesterol to plasma apolipoprotein A1 (Apo A1) in RCT pathway. A modest but statistically significant elevation in the mRNA level of (ABCA1) was found in the TMAO-treated peritoneal macrophages from C57BL/6J mice (Koeth et al. 2013). However, Mohammadi et al. (2016) observed elevations in both protein and mRNA levels of ABCA1 after $8 \mathrm{~h}$ of TMAO treatment in murine macrophage J774A.1 cell line, but no obvious alterations and significant decreases in ABCA1 expression were reported after $18 \mathrm{~h}$ and $24 \mathrm{~h}$ of TMAO treatment, respectively. In RAW264.7 macrophage, TMAO increased ABCA1-dependent cholesterol efflux to Apo A1, suggesting that the alteration in macrophage ABCA1 expression may not account for the TMAO-induced decline in RCT (Koeth et al. 2013). Moreover, TMAO conspicuously down-regulated the expression of CYP7A1, the rate-limiting enzyme in de novo biosynthesis of bile acid from cholesterol, as well as multiple he- patic transporters (including Oat1, Oat4, Mrp2 and Ntcp) for bile efflux from liver into intestine (Koeth et al. 2013). A significant smaller total bile acid pool size was observed in high-TMAO- or high-choline-fed $\mathrm{ApoE}^{--}$mice than that of control (Koeth et al. 2013; Chen et al. 2016). As a result, less cholesterol is likely to be eliminated via bile, leading to hepatic cholesterol accumulation and atherogenesis.

High plasma cholesterol level, in particular that of low-densitylipoprotein cholesterol (LDL-C), is believed to be a major risk factor for atherosclerosis. Inconsistent results regarding the TMAOrelated changes in plasma lipids have been reported. Some studies have shown that plasma total cholesterol (TC) and triacylglycerol (TG) levels were not affected by elevated plasma TMAO concentration in mice (Wang et al. 2011; Koeth et al. 2013; Hu et al. 2015; Seldin et al. 2016; Zhao et al. 2018). However, obvious increases in plasma TC or/and TG were observed in mice fed choline, a dietary precursor of TMAO, in some other studies (Chen et al. 2016; Ren et al. 2016). While declines in plasma TC and TG were reported in high-fat-fed $\mathrm{C} 57 \mathrm{BL} / 6 \mathrm{~J}$ mice administrated with $0.2 \%$ of TMAO in the diet (Gao et al. 2014).

\section{Potential dietary approaches for the prevention of TMAO- induced atherosclerosis}

Production of TMAO from dietary TMA-containing nutrients relies on both gut microbial activities in the intestine and host FMO processing in the liver. These multistep microbial-host interactions for TMAO generation might become potential therapeutic targets to reduce the risk of TMAO-associated CVD.

\subsection{Modulation of gut microbial composition by probiotics and/ or prebiotics}

Due to the critical role of gut microbes in the TMAO generation pathway, modulating intestinal microbiota composition can be a promising strategy to limit the liberation of TMA from dietary precursors. Probiotics are the alive microorganisms conferring a health benefit on host when administered in adequate amount (Sánchez et al. 2017). It was reported that probiotics could remodel intestinal microbial composition, and show protective effects in nonalcoholic fatty liver disease, obesity, diabetes, and intestinal diseases (Li et al. 2003; Delzenne et al. 2011; Gomes et al. 2014; Sánchez et al. 2017). Administration of probiotics might be able to reduce the microbiota-dependent production of TMA and TMAO. However, their effect on TMAO generation remains inconclusive. Martin et al. (2008) found that probiotic Lactobacillus paracasei supplement could decrease TMA concentration with TMAO being unchanged in the liver of mice. Meanwhile administration of another probiotics, Lactobacillus rhamnosus, raised hepatic TMAO concentrations without affecting hepatic TMA levels (Martin et al. 2008). In contrast, the multi-strain probiotic VSL\#3 (900 billion alive bacteria/day) did not influence the plasma TMAO levels, which was elevated after 4 weeks of feeding a high-fat diet in nineteen healthy, non-obese males (Boutagy et al. 2015). Similarly, 12 weeks of three times daily $6.5 \times 10^{9}$ colony-forming units (CFU) Lactobacillus casei Shirota $(L c S)$ administration did not alter plasma TMAO levels in patients with metabolic syndrome (Tripolt et al. 2015).

Dietary intervention with prebiotics is another possible strategy to modulate intestinal microbiota composition and reduce their TMA-generating capacity. Prebiotics is defined as a selectively 
fermented ingredient that can cause specific changes in the composition and/or activity of the gastrointestinal microbiota, thus conferring benefits upon host health (Valcheva and Dieleman 2016). However, the function of prebiotics in the microbiota-dependent production of TMA and TMAO is largely unknown. Chen et al. (2016) reported that resveratrol, a natural phytoalexin, helped to decease plasma TMA and TMAO levels, accompanied by development of smaller aortic atherosclerotic lesions in high-choline-fed mice. Analyses on the gut bacterial composition of mice revealed that resveratrol administration decreased the proportion of Firmicutes while it increased the proportion of Bacteroidetes (Chen et al. 2016). Moreover, resveratrol raised the relative abundances of the probiotics Lactobacillus and Bifidobacterium, resulted in elevations in the bile salt hydrolase activity, and enhancement in bile acid deconjugation and excretion in the feces (Chen et al. 2016). All these results suggest that resveratrol could be a beneficial prebiotic to alleviate the TMAO-induced atherosclerosis, in part, via gut microbiota remodeling.

Dietary probiotics and prebiotics can be promising candidates to modulate gut microbial composition and decrease the TMAO generation from dietary precursors thus preventing the development of TMAO-associated CVD. Future studies are required to investigate the effects of individual probiotic species on the TMAO generation pathway, the dose of probiotics/prebiotics used, and the duration of intervention needed to remodel the microbial composition in the host intestine.

\subsection{Regulation of host metabolism by functional foods and nu- traceuticals}

Functional foods possess a specific health-benefit beyond their nutritional properties when consumed regularly, while nutraceuticals are the bioactive agents derived from foods and can be used as diet supplements for health promotion (Espin et al. 2007). To date, functional foods and nutraceuticals have been used in the prevention and treatment of atherosclerosis and CVD, especially for those people who are in the marginal risk but do not warrant medical prescriptions. Application of functional foods or nutraceuticals may potentially regulate the host metabolism and attenuate the TMAOcorrelated progression of CVD. Nevertheless, research concerning the benefits of functional foods or nutraceuticals on TMAO-related adverse health consequences is very limited. Tartary buckwheat flavonoids were reported to alleviate the TMAO-induced reactive oxygen species (ROS) generation, vascular endothelial injury and liver oxidative damage in Kunming mice given a drinking water containing $1.5 \%$ TMAO (Hu et al. 2015). In the similar animal model given a drinking water containing $3 \%$ choline, phloretin, a natural polyphenol, exerted systemic benefits by mitigating the high-choline-induced dyslipidemia and hyperglycemia, relieving oxidative stress, attenuating endothelial dysfunction and liver injury (Ren et al. 2016). Of interest, marine fish intake was able to protect against CVD development due to its high content of $\omega-3$ polyunsaturated fatty acid (PUFAs), particularly eicosapentaenoic acid (EPA) and docosahexaenoic acid (DHA) (Kris-Etherton et al. 2002), whereas marine fish, rich in TMAO, may be detrimental to cardiovascular health. In the study of Gao et al. (2015), $2 \%$ fish oil in diet ameliorated the TMAO-exacerbated glucose intolerance, and inflammation in adipose tissue as well as in blood vessels in mice fed a high-fat-diet. As previously discussed, apart from its microbial remodeling capacity, resveratrol also helps to upregulate the hepatic CYP7A1 expression, promote hepatic bile acid de novo synthesis and compensate for the TMAO-induced reduction in fecal bile acid excretion (Chen et al. 2016). In addition, allicin, an or- ganic sulfur-containing compound from fresh garlic, was reported to reduce the production of TMAO from L-carnitine in C57BL/6 mice during a L-carnitine challenge test via modulating the gut microbiota (Wu et al. 2015).

Targeting at hepatic FMOs is another approach to restrict the conversion of TMAO from TMA. However, accumulation of TMA could lead to annoying trimethylaminuria or fish-odor-syndrome, which makes this strategy not practical and attractive. In this connection, exploitation of functional foods or nutraceuticals may be applied as novel preventive and therapeutic strategies for the TMAO-related CVD, but future studies are urgently required.

\section{Conclusion}

Blood TMAO has recently been identified as an independent marker for CVD. Most blood TMAO is derived from dietary TMAcontaining nutrients, relying on both intestinal gut microbes and host hepatic FMOs. Studies have shown that TMAO can promote atherogenesis mainly by exacerbating vascular inflammation, impairing vascular function and disturbing cholesterol homeostasis Dietary interventions by using probiotics and/or prebiotics, as well as functional foods and nutraceuticals may offer promising strategies for the prevention and treatment of TMAO-associated CVD. However, solid evidences are still lacking. There is a pressing need to investigate the functions of these beneficial dietary components in the production of TMAO and the TMAO-induced atherogenesis.

\section{References}

Bae, S., Ulrich, C.M., Neuhouser, M.L., Malysheva, O., Bailey, L.B., Xiao, L., Brown, E.C., Cushing-Haugen, K.L., Zheng, Y., Cheng, T.Y.D., Miller, J.W., Green, R., Lane, D.S., Beresford, S.A.A., and Caudill, M.A. (2014). Plasma choline metabolites and colorectal cancer risk in the Women's Health Initiative Observational Study. Cancer Res. 74(24): 7442-7452.

Bain, M.A., Fornasini, G., and Evans, A.M. (2005). Trimethylamine: Metabolic, pharmacokinetic and safety aspects. Curr. Drug Metab. 6(3): 227-240

Baker, R.G., Hayden, M.S., and Ghosh, S. (2011). NF-kB, inflammation, and metabolic disease. Cell Metab. 13(1): 11-22.

Benjamin, E.J., Blaha, M.J., Chiuve, S.E., Cushman, M., Das, S.R., Deo R., de Ferranti, S.D., Floyd, J., Fornage, M., Gillespie, C., Isasi, C.R., Jiménez, M.C., Jordan, L.C., Judd, S.E., Lackland, D., Lichtman, J.H., Lisabeth, L., Liu, S., Longenecker, C.T., Mackey, R.H., Matsushita, K., Mozaffarian, D., Mussolino, M.E., Nasir, K., Neumar, R.W., Palaniappan, L., Pandey, D.K., Thiagarajan, R.R., Reeves, M.J., Ritchey, M., Rodriguez, C.J., Roth, G.A., Rosamond, W.D., Sasson, C., Towfighi, A., Tsao, C.W., Turner, M.B., Virani, S.S., Voeks, J.H., Willey, J.Z., Wilkins, J.T., Wu, J.H., Alger, H.M., Wong, S.S., Muntner, P., and American Heart Association Statistics Committee and Stroke Statistics Subcommittee. (2017). Heart disease and stroke statistics-2017 update: A report from the American Heart Association. Circulation 135(10): e146-e603.

Bennett, B.J., de Aguiar Vallim, T.Q., Wang, Z., Shih, D.M., Meng, Y., Greg ory, J., Allayee, H., Lee, R., Graham, M., Crooke, R., Edwards, P.A. Hazen, S.L., and Lusis, A.J. (2013). Trimethylamine-N-oxide, a metabolite associated with atherosclerosis, exhibits complex genetic and dietary regulation. Cell Metab. 17(1): 49-60.

Bidulescu, A., Chambless, L.E., Siega-Riz, A.M., Zeisel, S.H., and Heiss, G. (2007). Usual choline and betaine dietary intake and incident coronary heart disease: the Atherosclerosis Risk in Communities (ARIC) Study. BMC Cardiovasc. Disord. 7: 20.

Boini, K.M., Hussain, T., Li, P.L., and Koka, S. (2017). Trimethylamine-NOxide instigates NLRP3 inflammasome activation and endothelial dysfunction. Cell Physiol. Biochem. 44(1): 152-162. 
Boutagy, N.E., Neilson, A.P., Osterberg, K.L., Smithson, A.T., Englund, T.R., Davy, B.M., Hulver, M.W., and Davy, K.P. (2015). Probiotic supplementation and trimethylamine- $\mathrm{N}$-oxide production following a high-fat diet. Obesity. 23(12): 2357-2363.

Chen, M.L., Yi, L., Zhang, Y., Zhou, X., Ran, L., Yang, J., Zhu, J.D., Zhang, Q.Y., and Mi, M.T. (2016). Resveratrol attenuates trimethylamine-Noxide (TMAO)-induced atherosclerosis by regulating TMAO synthesis and bile acid metabolism via remodeling of the gut microbiota. mBio. 7(2): e02210-15.

Chen, M.L., Zhu, X.H., Ran, L., Lang, H.D., Yi, L., and Mi, M.T. (2017). Trimethylamine- $\mathrm{N}$-oxide induces vascular inflammation by activating the NLRP3 inflammasome through the SIRT3-SOD2-mtROS signaling pathway. J. Am. Heart. Assoc. 6(9): e006347.

Cho, C.E., Taesuwan, S., Malysheva, O.V., Bender, E., Tulchinsky, N.F., Yan, J., Sutter, J.L., and Caudill, M.A. (2017). Trimethylamine-N-oxide (TMAO) response to animal source foods varies among healthy young men and is influenced by their gut microbiota composition: A randomized controlled trial. Mol. Nutr. Food Res. 61(1): 1600324.

Collins, H.L., Drazul-Schrader, D., Sulpizio, A.C., Koster, P.D., Williamson, Y., Adelman, S.J., Owen, K., Sanli, T., and Bellamine, A. (2016). L-Carnitine intake and high trimethylamine $\mathrm{N}$-oxide plasma levels correlate with low aortic lesions in ApoE(-/-) transgenic mice expressing CETP. Atherosclerosis. 244: 29-37.

Craciun, S., and Balskus, E.P. (2012). Microbial conversion of choline to trimethylamine requires a glycyl radical enzyme. Proc. Natl. Acad. Sci. U.S.A. 109(52): 21307-21312.

Delzenne, N.M., Neyrinck, A.M., Bäckhed, F., and Cani, P.D. (2011). Targeting gut microbiota in obesity: effects of prebiotics and probiotics. Nat. Rev. Endocrinol. 7(11): 639-646.

DiNicolantonio, J.J., Lavie, C.J., Fares, H., Menezes, A.R., and O'Keefe, J.H. (2013). L-carnitine in the secondary prevention of cardiovascular disease: systematic review and meta-analysis. Mayo Clin. Proc. 88(6): 544-551.

Dolphin, C.T., Janmohamed, A., Smith, R.L., Shephard, E.A., and Phillips, L.R. (1997). Missense mutation in flavin-containing mono-oxygenase 3 gene, FMO3, underlies fish-odour syndrome. Nat. Genet. 17(4): 491-494.

Espin, J.C., García-Conesa, M.T., and Tomás-Barberán, F.A. (2007). Nutraceuticals: Facts and fiction. Phytochemistry. 68(22-24): 2986-3008.

Fukami, K., Yamagishi, S.I., Sakai, K., Kaida, Y., Yokoro, M., Ueda, S., Wada, Y., Takeuchi, M., Shimizu, M., Yamazaki, H., and Okuda, S. (2015) Oral L-carnitine supplementation increases trimethylamine- $\mathrm{N}$-oxide but reduces markers of vascular injury in hemodialysis patients. J. Cardiovasc. Pharmacol. 65(3): 289-295.

Gao, X., Liu, X., Xu, J., Xue, C., Xue, Y., and Wang, Y. (2014). Dietary trimethylamine $\mathrm{N}$-oxide exacerbates impaired glucose tolerance in mice fed a high fat diet. J. Biosci. Bioeng. 118(4): 476-481.

Gao, X., Xu, J., Jiang, C., Zhang, Y., Xue, Y., Li, Z., Wang, J., Xue, C., and Wang, Y. (2015). Fish oil ameliorates trimethylamine N-oxide-exacerbated glucose intolerance in high-fat diet-fed mice. Food Funct. 6(4): 1117-1125.

Geng, J., Yang, C., Wang, B., Zhang, X., Hu, T., Gu, Y., and Li, J. (2017). Trimethylamine $\mathrm{N}$-oxide promotes atherosclerosis via CD36-dependent MAPK/JNK pathway. Biomed. Pharmacother. 97: 941-947.

Gillett, M.B., Suko, J.R., Santoso, F.O., and Yancey, P.H. (1997). Elevated levels of trimethylamine oxide in muscles of deep-sea gadiform teleosts: A high-pressure adaptation? J. Exp. Zool. 279(4): 386-391.

Gomes, A.C., Bueno, A.A., de Souza, R.G.M., and Mota, J.F. (2014). Gut microbiota, probiotics and diabetes. Nutr. J. 13: 60.

He, Z., and Chen, Z.Y. (2017). What are missing parts in the research story of trimethylamine- N-oxide (TMAO)? J. Agric. Food Chem. 65(26): 5227-5228.

Hernandez, D., Janmohamed, A., Chandan, P., Phillips, I.R., and Shephard, E.A. (2004). Organization and evolution of the flavin-containing monooxygenase genes of human and mouse: identification of nove gene and pseudogene clusters. Pharmacogenetics. 14(2): 117-130.

Hu, Y., Zhao, Y., Yuan, L., and Yang, X. (2015). Protective effects of tartary buckwheat flavonoids on high TMAO diet-induced vascular dysfunction and liver injury in mice. Food Funct. 6(10): 3359-3372.

Ke, Y., Li, D., Zhao, M., Liu, C., Liu, J., Zeng, A., Shi, X., Cheng, S., Pan, B., Zheng, L., and Hong, H. (2018). Gut flora-dependent metabolite trimethylamine- $\mathrm{N}$-oxide accelerates endothelial cell senescence and vascular aging through oxidative stress. Free Radic. Biol. Med. 116: 88-100.

Kelly, R.H., and Yancey, P.H. (1999). High contents of trimethylamine oxide correlating with depth in deep-sea teleost fishes, skates, and decapod crustaceans. Biol. Bull. 196(1): 18-25.

Koeth, R.A., Levison, B.S., Culley, M.K., Buffa, J.A., Wang, Z., Gregory, J.C. Org, E., Wu, Y., Li, L., Smith, J.D., Tang, W.H.W., DiDonato, J.A., Lusis, A.J., and Hazen, S.L. (2014). $\gamma$-Butyrobetaine is a proatherogenic intermediate in gut microbial metabolism of L-carnitine to TMAO. Cell Metab. 20(5): 799-812.

Koeth, R.A., Wang, Z., Levison, B.S., Buffa, J.A., Org, E., Sheehy, B.T., Britt, E.B., Fu, X., Wu, Y., Li, L., Smith, J.D., DiDonato, J.A., Chen, J., Li, H., Wu, G.D., Lewis, J.D., Warrier, M., Brown, J.M., Krauss, R.M., Tang, W.H.W., Bushman, F.D., Lusis, A.J., and Hazen, S.L. (2013). Intestinal microbiota metabolism of L-carnitine, a nutrient in red meat, promotes atherosclerosis. Nat. Med. 19(5): 576-585.

Koukouritaki, S.B., Simpson, P., Yeung, C.K., Rettie, A.E., and Hines, R.N. (2002). Human hepatic flavin-containing monooxygenases 1 (FMO1) and 3 (FMO3) developmental expression. Pediatr. Res. 51(2): 236243.

Kris-Etherton, P.M., Harris, W.S., and Appel, L.J. (2002). Fish consumption, fish oil, omega-3 fatty acids, and cardiovascular disease. Circulation 106: 2747-2757.

Lever, M., George, P.M., Slow, S., Bellamy, D., Young, J.M., Ho, M., McEntyre, C.J., Elmslie, J.L., Atkinson, W., Molyneux, S.L., Troughton, R.W., Frampton, C.M., Richards, A.M., and Chambers, S.T. (2014). Betaine and trimethylamine- $\mathrm{N}$-oxide as predictors of cardiovascular outcomes show different patterns in diabetes mellitus: An observational study. PLoS One. 9(12): e114969.

Li, Z., Yang, S., Lin, H., Huang, J., Watkins, P.A., Moser, A.B., Desimone, C., Song, X.Y., and Diehl, A.M. (2003). Probiotics and antibodies to TNF inhibit inflammatory activity and improve nonalcoholic fatty liver disease. Hepatology. 37(2): 343-350.

Lusis, A.J. (2000). Atherosclerosis. Nature. 407(6801): 233-241.

Ma, G., Pan, B., Chen, Y., Guo, C., Zhao, M., Zheng, L., and Chen, B. (2017). Trimethylamine $\mathrm{N}$-oxide in atherogenesis: impairing endothelia Self-repair capacity and enhancing monocyte adhesion. Biosci. Rep. 37(2): BSR20160244.

Mafune, A., Iwamoto, T., Tsutsumi, Y., Nakashima, A., Yamamoto, I., Yokoyama, K., Yokoo, T., and Urashima, M. (2016). Associations among serum trimethylamine-N-oxide (TMAO) levels, kidney function and infarcted coronary artery number in patients undergoing cardiovascular surgery: a cross-sectional study. Clin. Exp. Nephrol. 20(5): 731-739.

Martin, F.P.J., Wang, Y., Sprenger, N., Yap, I.K.S., Lundstedt, T., Lek, P., Rezzi, S., Ramadan, Z., van Bladeren, P., Fay, L.B., Kochhar, S., Lindon, J.C. Holmes, E., and Nicholson, J.K. (2008). Probiotic modulation of symbiotic gut microbial-host metabolic interactions in a humanized microbiome mouse model. Mol. Syst. Biol. 4(1): 157.

Mente, A., Chalcraft, K., Ak, H., Davis, A.D., Lonn, E., Miller, R., Potter, M.A., Yusuf, S., Anand, S.S., and McQueen, M.J. (2015). The relationship between trimethylamine-N-oxide and prevalent cardiovascular disease in a multiethnic population living in Canada. Can. J. Cardiol. 31(9): 1189-1194.

Millard, H.R., Musani, S.K., Dibaba, D.T., Talegawkar, S.A., Taylor, H.A., Tucker, K.L., and Bidulescu, A. (2016). Dietary choline and betaine; associations with subclinical markers of cardiovascular disease risk and incidence of CVD, coronary heart disease and stroke: the Jackson Heart Study. Eur. J. Nutr. 57(1): 51-60.

Miller, C.A., Corbin, K.D., da Costa, K.A., Zhang, S., Zhao, X., Galanko, J.A., Blevins, T., Bennett, B.J., O'Connor, A., and Zeisel, S.H. (2014). Effect of egg ingestion on trimethylamine- $\mathrm{N}$-oxide production in humans: a randomized, controlled, dose-response study. Am. J. Clin. Nutr. 100(3): 778-786.

Mohammadi, A., Najar, A.G., Yaghoobi, M.M., Jahani, Y., and Vahabzadeh, Z. (2016). Trimethylamine-N-oxide treatment induces changes in the ATP-binding cassette transporter $\mathrm{A} 1$ and scavenger receptor A1 in murine macrophage J774A.1 cells. Inflammation. 39(1): 393-404.

Rader, D.J., Alexander, E.T., Weibel, G.L., Billheimer, J., and Rothblat, G.H. (2009). The role of reverse cholesterol transport in animals and hu- 
mans and relationship to atherosclerosis. J. Lipid Res. 50(Suppl): S189-194.

Randrianarisoa, E., Lehn-Stefan, A., Wang, X., Hoene, M., Peter, A., Heinzmann, S.S., Zhao, X., Königsrainer, I., Königsrainer, A., Balletshofer B., Machann, J., Schick, F., Fritsche, A., Häring, H.U., Xu, G., Lehmann, R., and Stefan, N. (2016). Relationship of Serum Trimethylamine Noxide (TMAO) levels with early atherosclerosis in humans. Sci. Rep. 6: 26745.

Ren, D., Liu, Y., Zhao, Y., and Yang, X. (2016). Hepatotoxicity and endothelial dysfunction induced by high choline diet and the protective effects of phloretin in mice. Food Chem. Toxicol. 94: 203-212.

Rohrmann, S., Linseisen, J., Allenspach, M., von Eckardstein, A., and Müller, D. (2016). Plasma concentrations of trimethylamine-N-oxide are directly associated with dairy food consumption and low-grade inflammation in a German adult population. J. Nutr. 146(2): 283-289.

Romano, K.A., Vivas, E.I., Amador-Noguez, D., and Rey, F.E. (2015). Intestinal microbiota composition modulates choline bioavailability from diet and accumulation of the proatherogenic metabolite trimethylamine-N-oxide. mBio. 6(2): e02481-14.

Ross, R. (1999). Atherosclerosis-an inflammatory disease. N. Engl. J. Med. 340(2): 115-126.

Sánchez, B., Delgado, S., Blanco-Míguez, A., Lourenço, A., Gueimonde, M., and Margolles, A. (2017). Probiotics, gut microbiota, and their influence on host health and disease. Mol. Nutr. Food Res. 61(1): 1600240.

Schmedes, M., Aadland, E.K., Sundekilde, U.K., Jacques, H., Lavigne, C., Graff, I.E., Eng, Ø., Holthe, A., Mellgren, G., Young, J.F., Bertram, H.C., Liaset, B., and Clausen, M.R. (2016). Lean-seafood intake decreases urinary markers of mitochondrial lipid and energy metabolism in healthy subjects: Metabolomics results from a randomized crossover intervention study. Mol. Nutr. Food Res. 60(7): 1661-1672.

Schugar, R.C., Shih, D.M., Warrier, M., Helsley, R.N., Burrows, A., Ferguson, D., Brown, A.L., Gromovsky, A.D., Heine, M., Chatterjee, A., Li, L., Li, X.S., Wang, Z., Willard, B., Meng, Y., Kim, H., Che, N., Pan, C., Lee, R.G., Crooke, R.M., Graham, M.J., Morton, R.E., Langefeld, C.D., Das, S.K., Rudel, L.L., Zein, N., McCullough, A.J., Dasarathy, S., Tang, W.H.W., Erokwu, B.O., Flask, C.A., Laakso, M., Civelek, M., Naga Prasad, S.V., Heeren, J., Lusis, A.J., Hazen, S.L., and Brown, J.M. (2017). The TMAO-producing enzyme flavin-containing monooxygenase 3 regulates obesity and the beiging of white adipose tissue. Cell Rep. 19(12): 2451-2461.

Seldin, M.M., Meng, Y., Qi, H., Zhu, W., Wang, Z., Hazen, S.L., Lusis, A.J., and Shih, D.M. (2016). Trimethylamine N-oxide promotes vascular inflammation through signaling of mitogen-activated protein kinase and nuclear factor-KB. J. Am. Heart. Assoc. 5(2): e002767.

Senthong, V., Li, X.S., Hudec, T., Coughlin, J., Wu, Y., Levison, B., Wang, Z., Hazen, S.L., and Tang, W. (2016a). Plasma trimethylamine Noxide, a gut microbe-generated phosphatidylcholine metabolite, is associated with atherosclerotic burden. J. Am. Coll. Cardiol. 67(22) 2620-2628.

Senthong, V., Wang, Z., Li, X.S., Fan, Y., Wu, Y., Tang, W.H.W., and Hazen, S.L. (2016b). Intestinal microbiota-generated metabolite trimethylamine- $\mathrm{N}$-oxide and 5-year mortality risk in stable coronary artery disease: The contributory role of intestinal microbiota in a COURAGElike patient cohort. J. Am. Heart. Assoc. 5(6): e002816.

Shih, D.M., Wang, Z., Lee, R., Meng, Y., Che, N., Charugundla, S., Qi, H., Wu, J., Pan, C., Brown, J.M., Vallim, T., Bennett, B.J., Graham, M., Hazen, S.L., and Lusis, A.J. (2015). Flavin containing monooxygenase 3 exerts broad effects on glucose and lipid metabolism and atherosclerosis. J. Lipid Res. 56(1): 22-37.

Svensson, B.G., Akesson, B., Nilsson, A., and Paulsson, K. (1994). Urinary excretion of methylamines in men with varying intake of fish from the baltic sea. J. Toxicol. and Environ. Health. 41(4): 411-420.

Taesuwan, S., Cho, C.E., Malysheva, O.V., Bender, E., King, J.H., Yan, J., Thalacker-Mercer, A.E., and Caudill, M.A. (2017). The metabolic fate of isotopically labeled trimethylamine-N-oxide (TMAO) in humans. J. Nutr. Biochem. 45: 77-82.

Tang, W.H.W., Wang, Z., Kennedy, D.J., Wu, Y., Buffa, J.A., Agatisa-Boyle, B., Li, X.S., Levison, B.S., and Hazen, S.L. (2015a). Gut microbiotadependent trimethylamine $\mathrm{N}$-oxide (TMAO) pathway contributes to both development of renal insufficiency and mortality risk in chronic kidney disease. Circ. Res. 116(3): 448-455.

Tang, W.H.W., Wang, Z., Levison, B.S., Koeth, R.A., Britt, E.B., Fu, X., Wu, Y., and Hazen, S.L. (2013). Intestinal microbial metabolism of phosphatidylcholine and cardiovascular risk. N. Engl. J. Med. 368(17) 1575-1584.

Tang, W.H.W., Wang, Z., Shrestha, K., Borowski, A.G., Wu, Y., Troughton, R.W., Klein, A.L., and Hazen, S.L. (2015b). Intestinal microbiota-dependent phosphatidylcholine metabolites, diastolic dysfunction, and adverse clinical outcomes in chronic systolic heart failure. J. Card. Fail. 21(2): 91-96.

Treberg, J.R., and Driedzic, W.R. (2002). Elevated levels of trimethylamine oxide in deep-sea fish: evidence for synthesis and intertissue physiological importance. J. Exp. Zool. 293(1): 39-45.

Tripolt, N.J., Leber, B., Triebl, A., Köfeler, H., Stadlbauer, V., and Sourij, H. (2015). Effect of Lactobacillus casei Shirota supplementation on trimethylamine- $\mathrm{N}$-oxide levels in patients with metabolic syndrome: An open-label, randomized study. Atherosclerosis. 242(1): 141-144

Trøseid, M., Ueland, T., Hov, J.R., Svardal, A., Gregersen, I., Dahl, C.P., Aakhus, S., Gude, E., Bjorndal, B., Halvorsen, B., Karlsen, T.H., Aukrust, P., Gullestad, L., Berge, R.K., and Yndestad, A. (2015). Microbiota-dependent metabolite trimethylamine- $\mathrm{N}$-oxide is associated with disease severity and survival of patients with chronic heart failure. J. Intern. Med. 277(6): 717-726.

Valcheva, R., and Dieleman, L.A. (2016). Prebiotics: Definition and protective mechanisms. Best Pract. Res. Clin. Gastroenterol. 30(1): 27-37.

Veeravalli, S., Karu, K., Scott, F., Fennema, D., Phillips, I.R., and Shephard E.A. (2017). Effect of flavin-containing monooxygenase (FMO) genotype, mouse strain and gender on trimethylamine $\mathrm{N}$-oxide production, plasma cholesterol concentration and an index of atherosclerosis. Drug Metab. Dispos. 46(1): 20-25.

Velasquez, M., Ramezani, A., Manal, A., and Raj, D.S. (2016). Trimethylamine $\mathrm{N}$-oxide: The good, the bad and the unknown. Toxins. 8(11): 326.

Wang, Z., Klipfell, E., Bennett, B.J., Koeth, R., Levison, B.S., Dugar, B., Feldstein, A.E., Britt, E.B., Fu, X., Chung, Y.M., Wu, Y., Schauer, P., Smith J.D., Allayee, H., Tang, W.H.W., DiDonato, J.A., Lusis, A.J., and Hazen, S.L. (2011). Gut flora metabolism of phosphatidylcholine promotes cardiovascular disease. Nature. 472(7341): 57-63.

Wang, Z., Tang, W.H.W., Buffa, J.A., Fu, X., Britt, E.B., Koeth, R.A., Levison, B.S., Fan, Y., Wu, Y., and Hazen, S.L. (2014). Prognostic value of choline and betaine depends on intestinal microbiota-generated metabolite trimethylamine-N-oxide. Eur. Heart J. 35(14): 904-910.

Warrier, M., Shih, D.M., Burrows, A.C., Ferguson, D., Gromovsky, A.D., Brown, A.L., Marshall, S., McDaniel, A., Schugar, R.C., Wang, Z., Sacks, J., Rong, X., Vallim, T.A., Chou, J., Ivanova, P.T., Myers, D.S., Brown, H.A., Lee, R.G., Crooke, R.M., Graham, M.J., Liu, X., Parini, P., Tontonoz, P., Lusis, A.J., Hazen, S.L., Temel, R.E., and Brown, J.M. (2015) The TMAO-generating enzyme flavin monooxygenase 3 is a central regulator of cholesterol balance. Cell Rep. 10(3): 326-338.

Wilson, P.W.F., D’Agostino, R.B., Levy, D., Belanger, A.M., Silbershatz, H. and Kannel, W.B. (1998). Prediction of coronary heart disease using risk factor categories. Circulation. 97(18): 1837-1847.

Withers, P., Hefter, G., and Pang, T. (1994). Role of urea and methylamines in buoyancy of elasmobranchs. J. Exp. Biol. 188(1): 175-189.

Wu, W.K., Panyod, S., Ho, C.T., Kuo, C.H., Wu, M.S., and Sheen, L.Y. (2015) Dietary allicin reduces transformation of L-carnitine to TMAO through impact on gut microbiota. J. Funct. Foods. 15: 408-417.

Yancey, P.H. (2005). Organic osmolytes as compatible, metabolic and counteracting cytoprotectants in high osmolarity and other stresses. J. Exp. Biol. 208: 2819-2830.

Zeisel, S.H., and Warrier, M. (2017). Trimethylamine N-oxide, the micro biome, and heart and kidney disease. Annu. Rev. Nutr. 37: 157-181.

Zhao, Y., Yang, N., Gao, J., Li, H., Cai, W., Zhang, X., Ma, Y., Niu, X., Yang, G., Zhou, X., and Li, Y. (2018). The effect of different L-carnitine administration routes on the development of atherosclerosis in ApoE knockout mice. Mol. Nutr. Food Res. 62(5): 1700299.

Zheng, Y., Li, Y., Rimm, E.B., Hu, F.B., Albert, C.M., Rexrode, K.M., Manson, J.E., and Qi, L. (2016). Dietary phosphatidylcholine and risk of allcause and cardiovascular-specific mortality among US women and men. Am. J. Clin. Nutr. 104(1): 173-180.

Zhu, W., Gregory, J.C., Org, E., Buffa, J.A., Gupta, N., Wang, Z., Li, L., Fu, X., 
Wu, Y., Mehrabian, M., Sartor, R.B., McIntyre, T.M., Silverstein, R.L., Tang, W.H.W., DiDonato, J.A., Brown, J.M., Lusis, A.J., and Hazen, S.L. (2016). Gut microbial metabolite TMAO enhances platelet hyperreactivity and thrombosis risk. Cell. 165(1): 111-124.
Zhu, Y., Jameson, E., Crosatti, M., Schäfer, H., Rajakumar, K., Bugg, T.D.H., and Chen, Y. (2014). Carnitine metabolism to trimethylamine by an unusual Rieske-type oxygenase from human microbiota. Proc. Natl. Acad. Sci. U.S.A. 111(11): 4268-4273. 